\title{
Magnetic plasmon resonances in nanostructured topological insulators for strongly enhanced light-MoS 2 interactions
}

\author{
Hua Lu (1)', Zengji Yue', Yangwu Li', Yinan Zhang ${ }^{3,4}$, Mingwen Zhang', Wei Zeng', Xuetao Gan', Dong Mao', \\ Fajun Xiao ${ }^{1}$, Ting Mei', Weiyao Zhao ${ }^{2}$, Xiaolin Wang${ }^{2}$, Min Gu $\mathbb{B}^{3}$ and Jianlin Zhao ${ }^{1}$
}

\begin{abstract}
Magnetic resonances not only play crucial roles in artificial magnetic materials but also offer a promising way for light control and interaction with matter. Recently, magnetic resonance effects have attracted special attention in plasmonic systems for overcoming magnetic response saturation at high frequencies and realizing high-performance optical functionalities. As novel states of matter, topological insulators (TIs) present topologically protected conducting surfaces and insulating bulks in a broad optical range, providing new building blocks for plasmonics. However, until now, high-frequency (e.g. visible range) magnetic resonances and related applications have not been demonstrated in TI systems. Herein, we report for the first time, to our knowledge, a kind of visible range magnetic plasmon resonances (MPRs) in TI structures composed of nanofabricated $\mathrm{Sb}_{2} \mathrm{Te}_{3}$ nanogrooves. The experimental results show that the MPR response can be tailored by adjusting the nanogroove height, width, and pitch, which agrees well with the simulations and theoretical calculations. Moreover, we innovatively integrated monolayer $\mathrm{MoS}_{2}$ onto a TI nanostructure and observed strongly reinforced light-MoS 2 interactions induced by a significant MPR-induced electric field enhancement, remarkable compared with TI-based electric plasmon resonances (EPRs). The $\mathrm{MoS}_{2}$ photoluminescence can be flexibly tuned by controlling the incident light polarization. These results enrich TI optical physics and applications in highly efficient optical functionalities as well as artificial magnetic materials at high frequencies.
\end{abstract}

\section{Introduction}

Artificially structured materials have been broadly used to excite strong magnetic responses for the generation of crucial counterintuitive phenomena, including negative refraction, invisible cloaking, superlensing, etc. ${ }^{1,2}$. To overcome the weak magnetism of natural materials at optical frequencies, metallic molecules (e.g., split rings)

\footnotetext{
Correspondence: Hua Lu (hualu@nwpu.edu.cn) or

Yinan Zhang (zhangyinan@jnu.edu.cn) or Jianlin Zhao (jlzhao@nwpu.edu.cn) ${ }^{1}$ MOE Key Laboratory of Material Physics and Chemistry under Extraordinary Conditions, and Shaanxi Key Laboratory of Optical Information Technology, School of Physical Science and Technology, Northwestern Polytechnical University, 710129 Xi'an, China

${ }^{2}$ Institute for Superconducting \& Electronic Materials and ARC Centre of Excellence in Future Low-Energy Electronics, University of Wollongong, North Wollongong, NSW 2500, Australia
}

Full list of author information is available at the end of the article with magnetic resonances induced by conduction current loops were proposed to construct artificial magnetic materials with negative refractive indices in the terahertz $(\mathrm{THz})$ and mid-infrared ranges ${ }^{3,4}$. However, transferring magnetic resonances to higher frequencies (especially in the visible range) is restricted by the magnetic response saturation and stringent nanofabrication requirements $\mathrm{s}^{5,6}$. Fortunately, magnetic resonances with the generation of displacement current loops were observed in specially designed plasmonic nanostructures, such as metallic nanopillar pairs ${ }^{6}$, tailored nanoclusters ${ }^{7}$, and particle-film systems $^{8,9}$. These magnetic plasmon resonances (MPRs) possess excellent capabilities for engineering magnetism, confining light at the nanoscale, and enhancing the optical field in the high-frequency range, thus contributing to promising applications in light manipulation, perfect

\section{(c) The Author(s) 2020}

\footnotetext{
(c) Open Access This article is licensed under a Creative Commons Attribution 4.0 International License, which permits use, sharing, adaptation, distribution and reproduction in any medium or format, as long as you give appropriate credit to the original author(s) and the source, provide a link to the Creative Commons license, and indicate if changes were made. The images or other third party material in this article are included in the article's Creative Commons license, unless indicated otherwise in a credit line to the material. If material is not included in the article's Creative Commons license and your intended use is not permitted by statutory regulation or exceeds the permitted use, you will need to obtain permission directly from the copyright holder. To view a copy of this license, visit http://creativecommons.org/licenses/by/4.0/.
} 
absorption, sensitive sensing and reinforced light-matter interactions ${ }^{6-10}$. To broaden the actual applications of MPRs, novel materials are currently highly desirable to open new doors for the generation and control of magnetic resonance behavior at optical frequencies. For example, graphene split rings were theoretically predicted to produce a magnetic resonant response stronger than traditional metallic structures, but their operating frequencies were limited to the far-infrared range ${ }^{11}$. Recently, topological insulators (TIs), as new quantum states of matter, have attracted wide attention in electronics, optics, and plasmonics ${ }^{12-21}$. TIs present unconventional conducting edge (or surface) states with topological protection caused by strong spin-orbit coupling of insulating bulk states, distinct from ordinary metals and insulators ${ }^{12,13}$. The time-reversal symmetry of edge (or surface) states with gapless Dirac fermions enables the avoidance of carrier backscattering from nonmagnetic impurities ${ }^{13,14}$. The topological edge state was first confirmed in mercury telluride two-dimensional (2D) quantum wells ${ }^{12}$. Afterward, topological surface states with exotic Dirac cones were discovered in threedimensional (3D) nanomaterials (e.g. $\mathrm{Sb}_{2} \mathrm{Te}_{3}, \mathrm{Bi}_{2} \mathrm{Te}_{3}$, and $\left.\mathrm{Bi}_{2} \mathrm{Se}_{3}\right)^{13,14}$. In 2019, thousands of materials were predicted to possess TI properties, paving a prospective path for quantum computing, spintronics, and devices with lower energy consumption ${ }^{22,23}$. Recently, $\mathrm{Bi}_{2} \mathrm{Te}_{3}$, $\mathrm{Bi}_{2} \mathrm{Se}_{3}$, and $\mathrm{Bi}_{1.5} \mathrm{Sb}_{0.5} \mathrm{Te}_{1.8} \mathrm{Se}_{1.2}$ TIs have been verified to display ultrahigh refractive indices and light-driven plasmonic activities in an ultrabroad optical range from ultraviolet (UV) to $\mathrm{THz}^{16-21}$. MPRs in TIs will be particularly useful for promoting the high-frequency optical activities of TIs and enriching their practical applications, especially in light-matter interactions. However, until now, MPRs and relevant applications have not been reported in TI systems.

Herein, we demonstrate for the first time, to our knowledge, a kind of visible range MPR effect in nanofabricated single-crystalline $\mathrm{Sb}_{2} \mathrm{Te}_{3}$ TI nanogrooves. The experimental results reveal that the MPR response has a particular dependence on the nanogroove height, width, and pitch, consistent with simulations and theoretical calculations. To explore actual applications of TI MPRs, we innovatively integrated monolayer $\mathrm{MoS}_{2}$ with a nanostructured TI to improve the intrinsically weak interactions between light and atomic-layer materials. Polarization-dependent photoluminescence (PL) emission was experimentally observed and reasonably analyzed. Benefitting from the strong MPR-induced electric field enhancement, the $\mathrm{MoS}_{2}$ PL intensity was remarkably reinforced compared with TI electric plasmon resonances (EPRs). These results will open a new door for exploring novel TI optical physics and applications in optoelectronic devices and artificial magnetic materials.

\section{Results}

Optical constant and nanostructure of the $\mathrm{Sb}_{2} \mathrm{Te}_{3}$ single crystal

As shown in Fig. 1a, the TI nanogroove grating structure is fabricated on the surface of a $\mathrm{Sb}_{2} \mathrm{Te}_{3}$ single-crystal film using the focused ion beam (FIB) milling method (see "Materials and methods" section). The height, width, and pitch of the nanogrooves are denoted by $h, d$, and $p$, respectively. As a primary member of the 3D TI family, the $\mathrm{Sb}_{2} \mathrm{Te}_{3}$ material possesses distinctly discrepant surface and bulk states, exhibiting excellent optical characteristics ${ }^{13,15}$. Here, the $\mathrm{Sb}_{2} \mathrm{Te}_{3}$ single crystal is grown using the melting and slow-cooling method (see "Materials and methods" section). To clarify the material morphology, we employ transmission electron microscopy (TEM) to obtain the selected area electron diffraction (SAED) pattern and a high-resolution TEM (HRTEM) image of the $\mathrm{Sb}_{2} \mathrm{Te}_{3}$ microflake. The $\mathrm{Sb}_{2} \mathrm{Te}_{3}$ microflake depicted in Fig. $1 \mathrm{~b}$ is fabricated through mechanical exfoliation and chemical etching methods (Supplementary Methods). As shown in Fig. 1c, the sharp diffraction spots and atomic lattice arrangement verify the hexagonal packed structure of the high-quality $\mathrm{Sb}_{2} \mathrm{Te}_{3}$ single crystal. The chemical composition is confirmed by energy-dispersive X-ray spectroscopy (EDS), revealing that the elemental molar ratio of Sb:Te is 2:3 (Supplementary Fig. S1). The high crystalline quality of $\mathrm{Sb}_{2} \mathrm{Te}_{3}$ can be further verified by the Raman spectrum (Supplementary Fig. S2). The complex relative permittivities of TI materials can be measured by a spectroscopic ellipsometer with considering the surface and bulk states ${ }^{17}$. The conducting surface and insulating bulk can be fitted with the Drude and Tauc-Lorentz dispersion formulas, respectively (Supplementary Methods). Figure 1d shows the fitted relative permittivity of singlecrystalline $\mathrm{Sb}_{2} \mathrm{Te}_{3}$ in the UV, visible, and near-infrared ranges, which agrees well with the experimental results. The surface and bulk permittivities $\left(\varepsilon_{\mathrm{s}}\right.$ and $\left.\varepsilon_{\mathrm{b}}\right)$ are depicted in Fig. 1e, f, respectively. The surface permittivity satisfies the conditions of $\operatorname{Re}\left(\varepsilon_{\mathrm{s}}\right)<0$ and $-\operatorname{Re}\left(\varepsilon_{\mathrm{s}}\right)>\operatorname{Im}\left(\varepsilon_{\mathrm{s}}\right)$ at wavelengths from 250 to $2065 \mathrm{~nm}$. This metal-like property of the surface state provides the possibility of generating the plasmonic response at high frequencies ${ }^{24-26}$. The negative permittivity of the bulk state at shorter wavelengths (from 253 to $760 \mathrm{~nm}$ ) can be attributed to the strong interband electronic absorption similar to semiconductors ${ }^{17}$. Thus, the bulk state can also contribute to the formation of plasmonic resonances at visible wavelengths of less than $760 \mathrm{~nm}$. The surface and bulk states together give rise to the negative permittivity of $\mathrm{Sb}_{2} \mathrm{Te}_{3}$ at wavelengths from 250 to $895 \mathrm{~nm}$, as shown in Fig. 1d. The fitting results (Supplementary Table S1) illustrate that the $\mathrm{Sb}_{2} \mathrm{Te}_{3}$ bulk possesses a bandgap of $\sim 0.33 \mathrm{eV}$, which is consistent with the reported $0.3 \mathrm{eV}^{27}$. The $\mathrm{Sb}_{2} \mathrm{Te}_{3}$ surface presents an ultrathin layer of $2.6 \mathrm{~nm}$ (i.e., $t=2.6 \mathrm{~nm}$ ), similar to the 

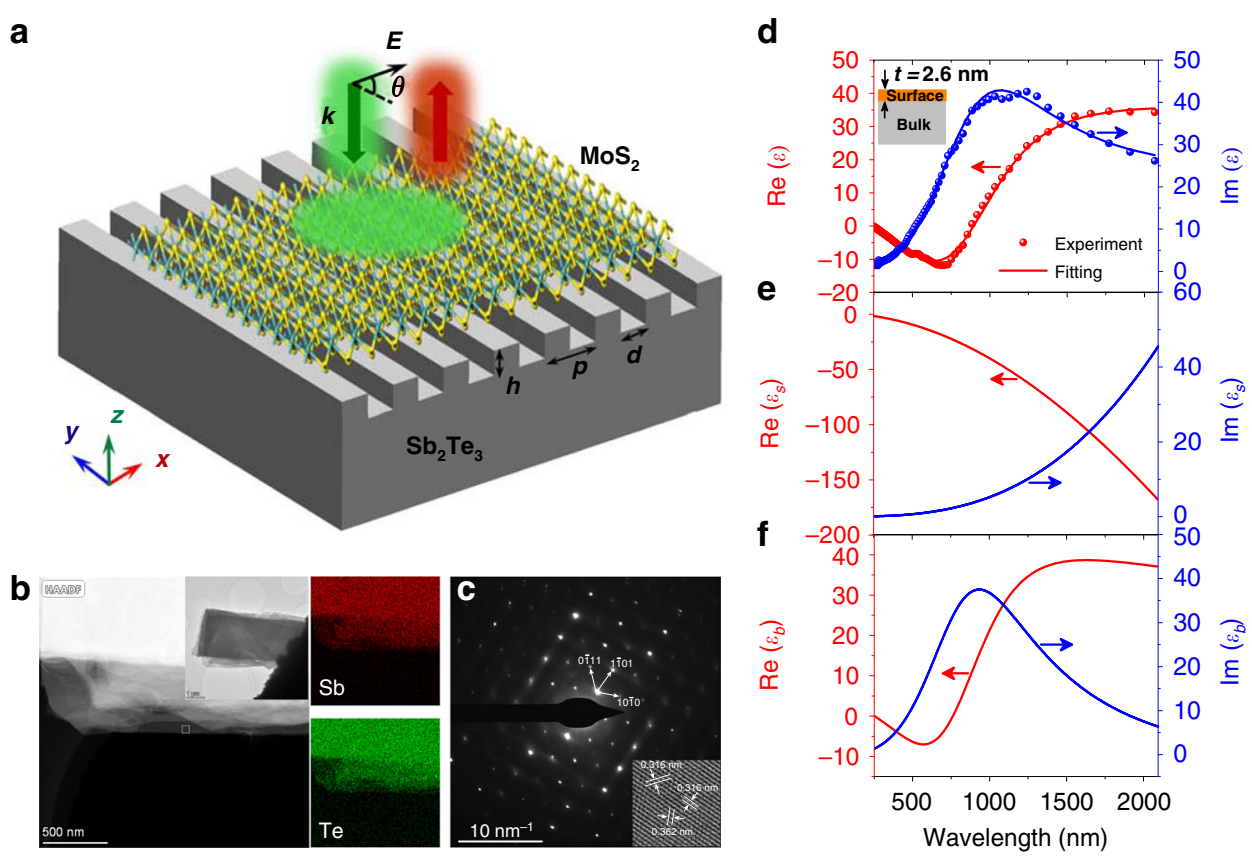

Fig. 1 TI nanostructure, material characterization, and optical constant. a Schematic of a $\mathrm{Sb}_{2} \mathrm{Te}_{3} \mathrm{TI}$ nanogroove grating with height $h$, width $d$, and pitch $p$ underneath monolayer $\mathrm{MoS}_{2}$. The light is incident on the nanostructure with a polarization angle of $\theta$. $\mathbf{b}$ TEM high-angle annular darkfield (HAADF) image of a part of a thin $\mathrm{Sb}_{2} \mathrm{Te}_{3}$ microflake (inset) on the carbon support film of a copper microgrid. The dotted rectangle denotes the area selected for electron diffraction and high-resolution TEM. The right images are the EDS mapping pictures of the measured part of the $\mathrm{Sb}_{2} \mathrm{Te}_{3}$ microflake for determining the spatial distributions of elements Sb and Te. c SAED pattern and HRTEM image (inset) of the $\mathrm{Sb}_{2} \mathrm{Te}_{3} \mathrm{single}_{\mathrm{c}} \mathrm{crystal}$. d Measured (circles) and fitted (curves) relative permittivities of the $\mathrm{Sb}_{2} \mathrm{Te}_{3}$ single crystal in the UV, visible, and near-infrared ranges. The fitted real and imaginary permittivities are obtained from the ellipsometer software with a layer-on-bulk model (inset) for the $\mathrm{Sb}_{2} \mathrm{Te}_{3} \mathrm{Tl}_{\mathrm{e}}$ e, $\mathbf{f}$ Real and imaginary permittivities of the conducting surface and insulating bulk.

reported $2.5 \mathrm{~nm}$ for the TIs in the same family ${ }^{28}$. To the best of our knowledge, this is the first report of surface and bulk optical constants for the $\mathrm{Sb}_{2} \mathrm{Te}_{3}$ single crystal, laying the foundation for exploring $\mathrm{Sb}_{2} \mathrm{Te}_{3}$ optical activities and functionalities.

\section{Magnetic plasmon resonances in $\mathrm{Sb}_{2} \mathrm{Te}_{3}$ nanostructures}

First, a nanogroove grating with $h=110 \mathrm{~nm}, d=$ $130 \mathrm{~nm}$, and $p=450 \mathrm{~nm}$ is fabricated on the $\mathrm{Sb}_{2} \mathrm{Te}_{3}$ film (with a thickness of $>300 \mathrm{~nm}$ ) mechanically exfoliated onto a Si substrate. Scanning electron microscopy (SEM) and atomic force microscopy (AFM) are used to measure the structural profile of the nanogrooves, as shown in Fig. 2a, b, respectively. The reflection spectra from the $\mathrm{Sb}_{2} \mathrm{Te}_{3}$ nanogroove grating are measured using a microspectrometer system (see "Materials and methods" section). The experimental results in Fig. 2c reveal that the incident light with polarization perpendicular to the nanogrooves (i.e., $\theta=0^{\circ}$ ) possesses a distinct reflection dip at $\sim 736 \mathrm{~nm}$. However, the reflection dip disappears when the polarization is parallel to the nanogrooves (i.e., $\theta=90^{\circ}$ ). The finite-difference time-domain (FDTD) numerical simulations (see "Materials and methods" section) agree well with the experimental results, as depicted in Fig. 2d. To clarify the reflection mechanism, we plot the magnetic and electric field distributions at the reflection dip when $\theta=0^{\circ}$, as shown in Fig. 2e, f, respectively. Interestingly, the magnetic field energies are enhanced and are mainly concentrated in the $\mathrm{Sb}_{2} \mathrm{Te}_{3}$ nanogrooves, exhibiting a strong diamagnetic effect. In Fig. 2f, the arrows indicate the direction and amplitude of the electric field vector, revealing the generation of displacement current loops in the nanogrooves. The electric current along the nanogroove surface is excited by the magnetic field component parallel to the $\mathrm{Sb}_{2} \mathrm{Te}_{3}$ nanogroove (Supplementary Fig. S3). The visible range resonance generated in this split-ring-like TI nanostructure is regarded as a typical magnetic resonance similar to $\mathrm{MPR}^{29}$. When the magnetic field component of incident light is perpendicular to the nanogrooves (i.e., $\theta=90^{\circ}$ ), the magnetic resonance cannot be effectively excited, restraining the appearance of the reflection dip. Under the resonant condition, the TI surface charges accumulate at the upper corners of the nanogrooves, accompanied by a strong electric field intensity $\left(\left|E / E_{\mathrm{i}}\right|^{2}\right)$ enhancement of $>200$-fold, as depicted in Fig. 2f. From Fig. 2c, we find that TIs can present a broader MPR (or MPR-like) spectrum than metals. The broad MPR spectrum contributes to a 
a
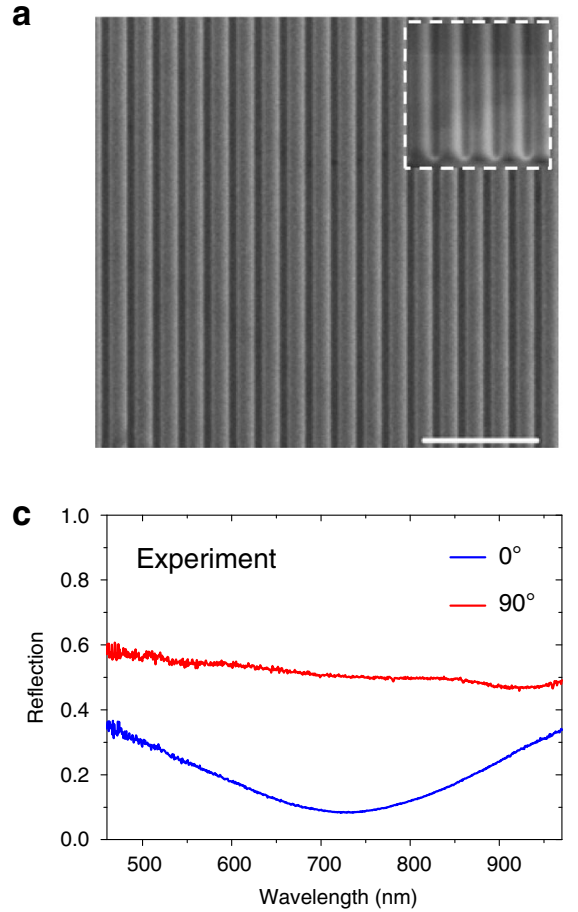

e

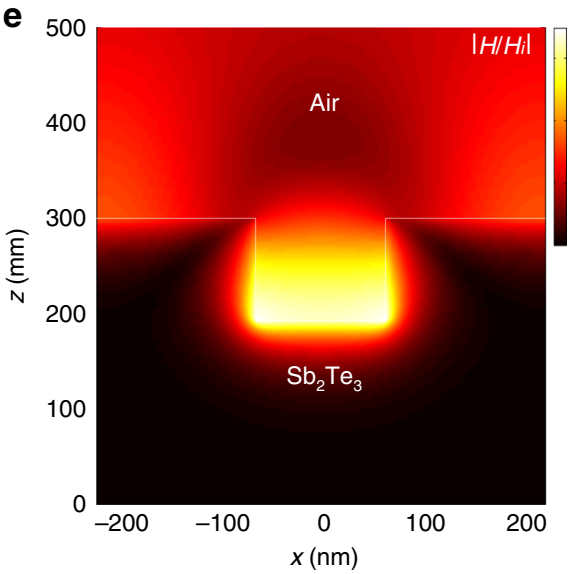

b
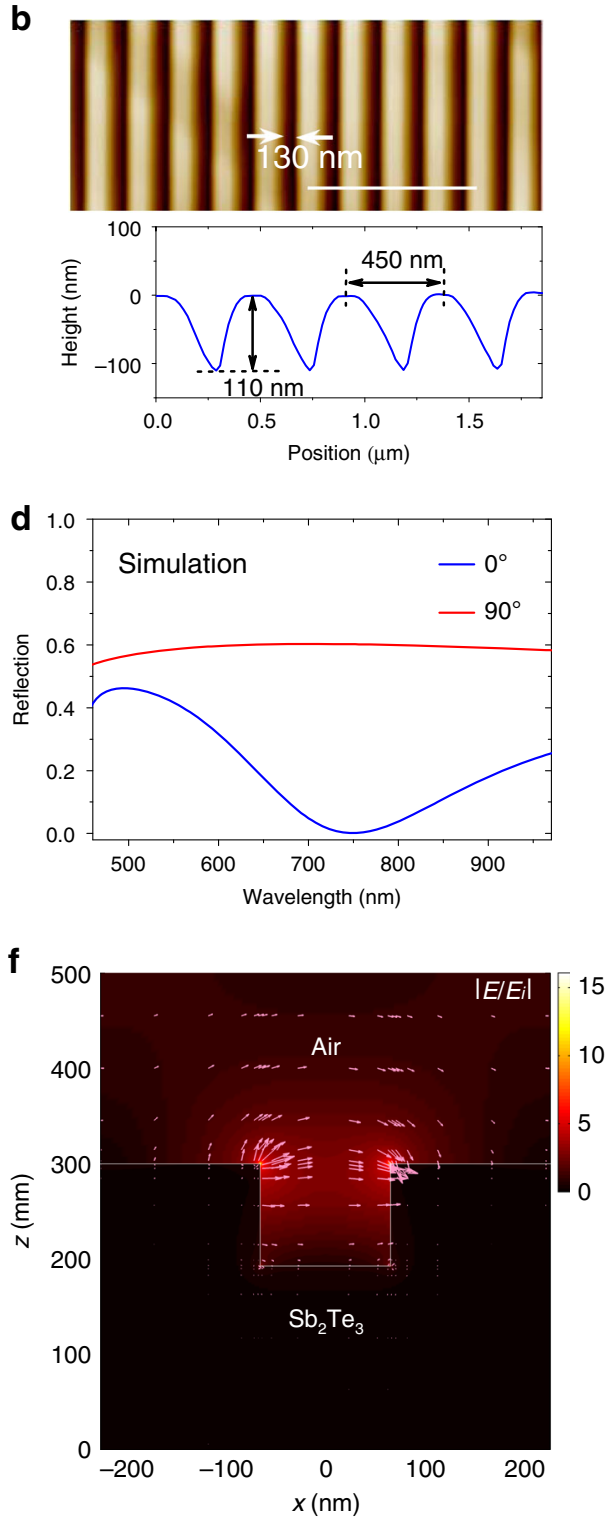

Fig. 2 Structure characterization, reflection spectra, and MPR field distribution of TI nanogrooves. a Top-view SEM image of the $\mathrm{Sb}_{2} \mathrm{Te}_{3}$ nanogroove grating with $h=110 \mathrm{~nm}, d=130 \mathrm{~nm}$, and $p=450 \mathrm{~nm}$. The scale bar is $2 \mu \mathrm{m}$. The inset shows an SEM image of $\mathrm{Sb}_{2} \mathrm{Te}_{3} \mathrm{nanogrooves}$ with a 52-degree view. $\mathbf{b}$ Corresponding AFM image of the $\mathrm{Sb}_{2} \mathrm{Te}_{3}$ nanogrooves and the nanogroove height profile along the white line. c, $\mathbf{d}$ Experimentally measured and numerically simulated reflection spectra from the $\mathrm{Sb}_{2} \mathrm{Te}_{3}$ nanogroove grating when $\theta=0^{\circ}$ and $90^{\circ}$. e Distribution of the magnetic field $\left|H / H_{i}\right|$ in a periodic unit of $\mathrm{Sb}_{2} \mathrm{Te}_{3}$ nanogrooves at the MPR wavelength when $\theta=0^{\circ}$. $\mathbf{f}$ Corresponding distribution of electric field $\left|E / E_{\mathrm{i}}\right|$. The arrows indicate the directions and relative magnitudes of the electric field. Here, $H_{\mathrm{i}}$ and $E_{\mathrm{i}}$ are the magnetic and electric field amplitudes of the incident light, respectively.

relatively large wavelength range for strong field enhancement. The significant field enhancement in nanostructured TIs will open a new door for the improvement of nanoscale light-matter interactions. It is worth noting that the TI surface layer contributes to the redshift and narrowing of the resonant spectrum ${ }^{17}$ (Supplementary Fig. S4a). At the MPR wavelength, the TI surface and bulk present absorption peak values of $\sim 6 \%$ and $\sim 94 \%$, respectively (Supplementary Fig. S4b).
Subsequently, we investigate the dependence of the magnetic resonance on the structural parameters (i.e., height $h$, width $d$, and pitch $p$ ) of the $\mathrm{Sb}_{2} \mathrm{Te}_{3}$ TI nanogrooves. Figure 3 a shows that the reflection dip presents a distinct redshift with increasing $h$ when $\theta=0^{\circ}$. The MPR wavelength has a nearly linear relationship with $h$ when $p=450 \mathrm{~nm}$ and $d=130 \mathrm{~nm}$, as depicted in Fig. 3b. The experimental results are in excellent agreement with the numerical simulations. The MPRs can be reasonably 

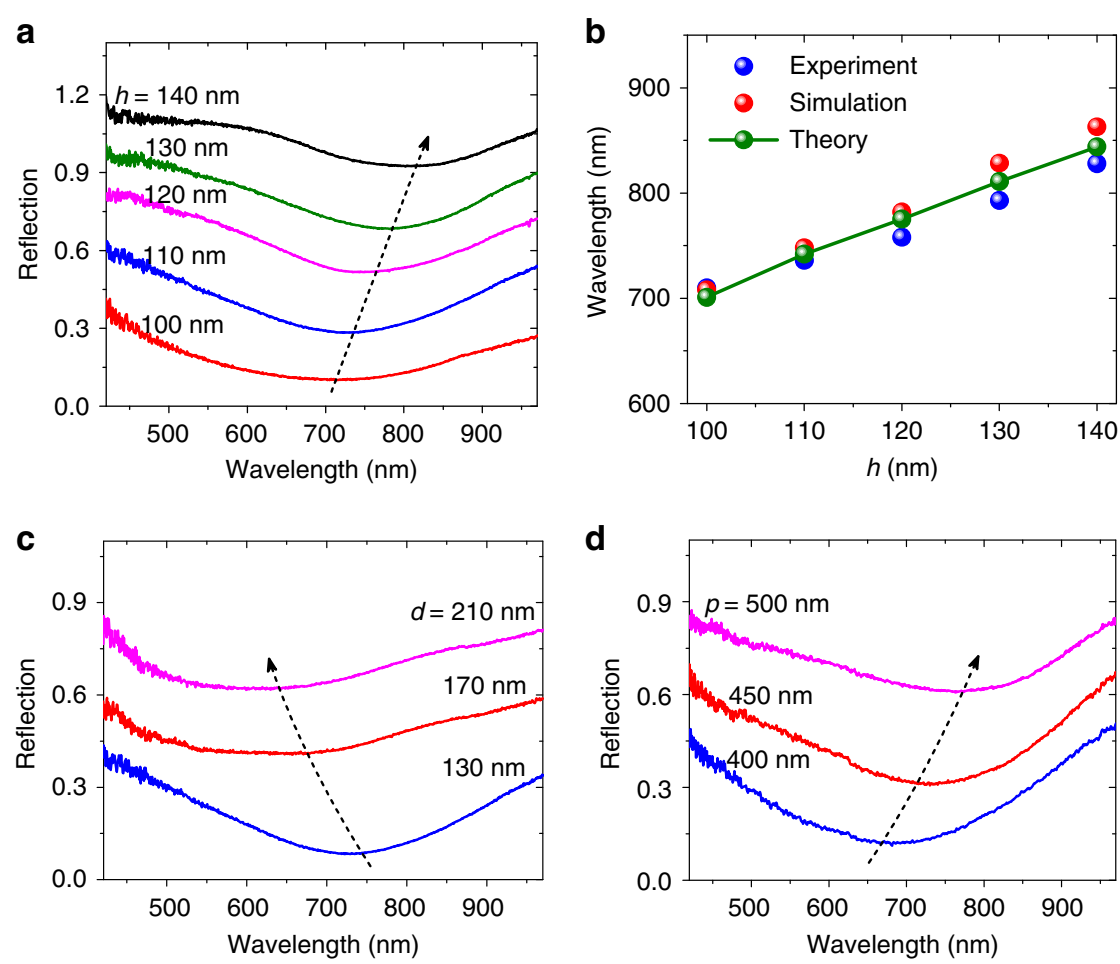

Fig. 3 Measured MPR spectral response of TI nanogrooves with different structural parameters. a Experimentally measured reflection spectra from the $\mathrm{Sb}_{2} \mathrm{Te}_{3}$ nanogroove grating with different heights $h=100,110,120,130$, and $140 \mathrm{~nm}$ when $d=130 \mathrm{~nm}, p=450 \mathrm{~nm}$, and $\theta=0^{\circ}$. b Corresponding MPR wavelengths of $\mathrm{Sb}_{2} \mathrm{Te}_{3}$ nanogrooves with different $h$. The experimental, simulation, and theoretical results are obtained by the micro-spectrometer, FDTD method, and MLC circuit model, respectively. c, d Experimentally measured reflection spectra from the $\mathrm{Sb}_{2} \mathrm{Te}_{3}$ nanogroove grating with different widths $d=130,170$, and $210 \mathrm{~nm}$, when $h=110 \mathrm{~nm}, p=450 \mathrm{~nm}$, and $\theta=0^{\circ}$ and with different pitches $p=400$, 450, and $500 \mathrm{~nm}$, when $h=120 \mathrm{~nm}, d=170 \mathrm{~nm}$, and $\theta=0^{\circ}$.

analyzed by a mutual inductor-inductor-capacitor (MLC) circuit model ${ }^{30}$. The MPR wavelength can be described as $\lambda_{M}=2 \pi c[(L+M) C]^{0.5}$, where $L, M$, and $C$ are the inductance containing the nanogroove ridge inductance/kinetic inductance, the mutual inductance between two adjacent circuits, and the capacitance between two nanogroove ridges (Supplementary Methods). According to the MLC circuit model, we can theoretically deduce the MPR wavelength in the $\mathrm{Sb}_{2} \mathrm{Te}_{3}$ nanogrooves. The theoretical calculations are well consistent with the experimental and simulation results, as shown in Fig. 3b. The MLC circuit model indicates that the MPR response is also dependent on the nanogroove width $d$. Here, we measure the reflection spectra from the $\mathrm{Sb}_{2} \mathrm{Te}_{3}$ nanogroove gratings with different $d$ when $h=$ $110 \mathrm{~nm}$ and $p=450 \mathrm{~nm}$. The experimental results in Fig. 3c demonstrate that the MPR wavelength exhibits a blueshift as $d$ increases, which agrees well with the simulations (Supplementary Fig. S5). We also find that the MPR response also depends on the pitch of the nanogroove grating. The experiments in Fig. $3 \mathrm{~d}$ show that the MPR wavelength redshifts with increasing $p$, in accordance with the simulations (Supplementary Fig. S5). From the MLC circuit model, we can see that the mutual inductance $M$ increases with $p$, giving rise to the redshift of $\lambda_{M}$. The nanogroove height, width, and pitch are the effective parameters for the tuning and selection of MPR wavelengths in TIs.

\section{$\mathrm{PL}$ emission from the monolayer $\mathrm{MoS}_{2} / \mathrm{Sb}_{2} \mathrm{Te}_{3}$ nanogroove heterostructure}

As mentioned above, the magnetic resonance in the TI nanostructure with strong field enhancement may offer a promising route for boosting light-matter interactions. Transition metal dichalcogenides (TMDCs), as a kind of 2D materials with unique semiconductor-like band structures, are regarded as a favorable platform for advancing next-generation optoelectronics due to the unique electric, mechanical and optical properties ${ }^{31,32}$. As a prototypical TMDC semiconductor, $\mathrm{MoS}_{2}$ possesses a state transition from an indirect bandgap of $1.2 \mathrm{eV}$ to a direct bandgap of $1.8 \mathrm{eV}$ as it transforms from bulk to monolayer ${ }^{31,33}$. The photoemission, excitonic binding, and chemical stability of monolayer $\mathrm{MoS}_{2}$ have drawn wide attention for the PL emission, promoting the achievement of atomically thin active light emitters and 
a

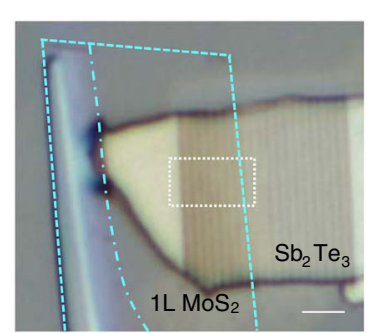

d

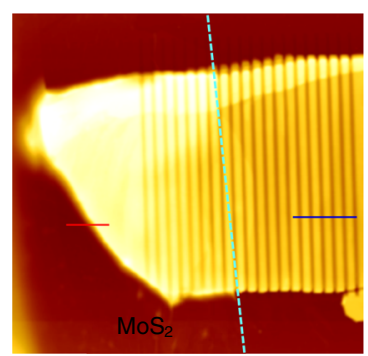

b
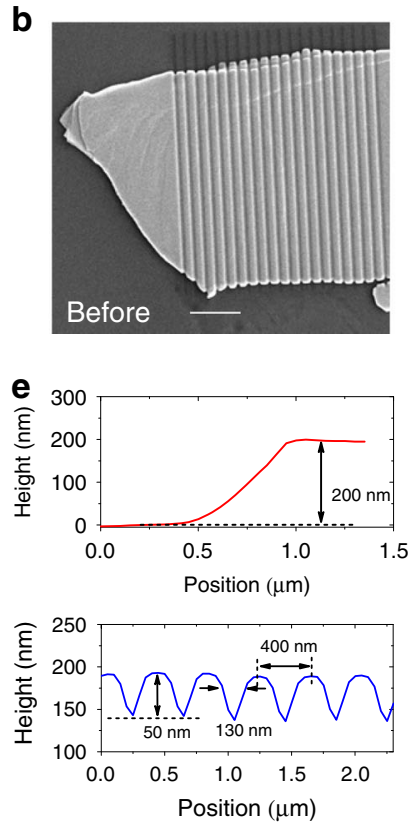

C

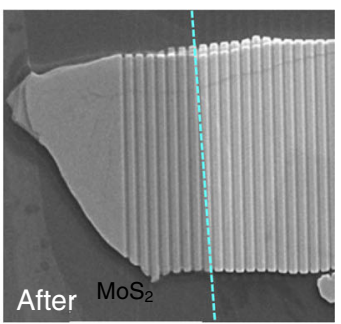

f

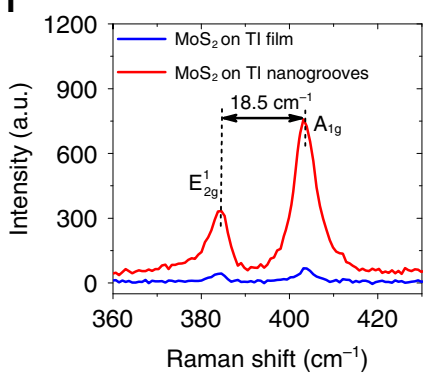

Fig. 4 Structure and material characterization of the monolayer $\mathrm{MoS}_{2} / \mathrm{Sb}_{2} \mathrm{Te}$ n nanogroove heterostructure. a Optical microscope image of $\mathrm{Sb}_{2} \mathrm{Te}_{3}$ nanogrooves with a transferred $\mathrm{MoS}_{2}$ layer. The area marked by the cyan dashed line denotes the $\mathrm{MoS}_{2}$ layer. The area on the right side of the dashed-dotted line represents monolayer $(1 \mathrm{~L}) \mathrm{MoS}_{2}$. The scale bar is $2 \mu \mathrm{m}$. b, c SEM images of $\mathrm{Sb}_{2} \mathrm{Te}_{3}$ nanogrooves before and after transferring $\mathrm{MoS}_{2}$. The scale bar is $2 \mu \mathrm{m}$ in $\mathbf{b}$. d AFM image of $\mathrm{Sb}_{2} \mathrm{Te}_{3}$ nanogrooves with $\mathrm{MoS}_{2}$. e AFM-measured height profiles of the $\mathrm{Sb}_{2} \mathrm{Te}_{3}$ film and nanogrooves at the positions along the red and blue lines in $\mathbf{d}$. $\mathbf{f}$ Raman spectra of the $\mathrm{MoS}_{2}$ layer on the $\mathrm{Sb}_{2} \mathrm{Te}_{3} \mathrm{TI}$ film and nanogrooves excited with the $532 \mathrm{~nm}$ laser when $\theta=0^{\circ}$.

sources ${ }^{33-35}$. However, the atomically thin layer with poor light-matter interactions hinders the substantial application of $\mathrm{MoS}_{2}$ for light harvesting and emission ${ }^{33,35}$. Here, we innovatively integrate a $\mathrm{MoS}_{2}$ atomic monolayer with a $\mathrm{Sb}_{2} \mathrm{Te}_{3}$ TI nanostructure to explore the tailoring and enhancement of PL emission. As depicted in Figs. 1a and $4 \mathrm{a}$, a $\mathrm{MoS}_{2}$ flake is mechanically exfoliated and coated on the $\mathrm{Sb}_{2} \mathrm{Te}_{3}$ nanogrooves using the fixed-point transfer method (see "Materials and methods" section). Figure 4b, c shows SEM images of the $\mathrm{Sb}_{2} \mathrm{Te}_{3}$ nanostructure before and after transferring the $\mathrm{MoS}_{2}$ flake, respectively. We can see that the $\mathrm{MoS}_{2}$ layer is precisely transferred onto the $\mathrm{Sb}_{2} \mathrm{Te}_{3}$ film with fabricated nanogrooves. The nanogroove grating is fabricated with $h=50 \mathrm{~nm}, d=130 \mathrm{~nm}$, and $p=$ $400 \mathrm{~nm}$ on the $\mathrm{Sb}_{2} \mathrm{Te}_{3}$ film with a thickness of $\sim 200 \mathrm{~nm}$, as shown in Fig. 4d, e. The Raman spectrum in Fig. 4f shows that the in-plane vibrational mode $E_{2 g}^{1}$ and out-ofplane vibrational mode $\mathrm{A}_{1 g}$ can be excited when a $532 \mathrm{~nm}$ laser beam impinges onto the $\mathrm{MoS}_{2}$ layer over $\mathrm{Sb}_{2} \mathrm{Te}_{3}$. The frequency difference between the $E_{2 g}^{1}$ and $\mathrm{A}_{1 g}$ modes is $\sim 18.5 \mathrm{~cm}^{-1}$, which agrees well with the reported value of monolayer $\mathrm{MoS}_{2}{ }^{34}$. As depicted in the inset of Fig. $5 \mathrm{a}$, the $\mathrm{Sb}_{2} \mathrm{Te}_{3}$ nanogroove grating with monolayer $\mathrm{MoS}_{2}$ presents a reflection dip at $\sim 532 \mathrm{~nm}$ when $\theta=0^{\circ}$ while retaining a high reflection when $\theta=90^{\circ}$. This illustrates that the magnetic resonance can be generated at $\sim 532 \mathrm{~nm}$ in the monolayer $\mathrm{MoS}_{2} / \mathrm{Sb}_{2} \mathrm{Te}_{3}$ nanogroove heterostructure. The numerical simulations are consistent with the experimental results (Supplementary Fig. S6). To reveal the light- $\mathrm{MoS}_{2}$ interaction enhancement, we demonstrate the luminescence emission response in the monolayer $\mathrm{MoS}_{2} / \mathrm{Sb}_{2} \mathrm{Te}_{3}$ nanogroove heterostructure using a confocal micro-spectrometry system with an excitation wavelength of $532 \mathrm{~nm}$. Figure 5 a displays the PL intensity spectra of the heterostructure for different incident polarization angles. Two PL peaks occur at 674.5 and $618.0 \mathrm{~nm}$ when $\theta=90^{\circ}$, as can be more clearly seen in Supplementary Fig. S7. The inset of Fig. 5b depicts the PL intensity map spectrally integrated from 665 to $695 \mathrm{~nm}$ in the dashed square frame of Fig. 4a when $\theta=0^{\circ}$. We can see that the $\mathrm{Sb}_{2} \mathrm{Te}_{3}$ nanogrooves with $\mathrm{MoS}_{2}$ can effectively excite the PL signal, while the $\mathrm{Sb}_{2} \mathrm{Te}_{3}$ nanogrooves cannot solely emit upon photoexcitation, as shown in Fig. $5 \mathrm{c}$. The above two PL peaks correspond to the positions of the $\mathrm{A}$ and $\mathrm{B}$ direct excitonic transitions for monolayer $\mathrm{MoS}_{2}$ at the $\mathrm{K}$ point of the Brillouin zone ${ }^{36}$. The two resonances derive from the energy splitting from spin-orbit coupling of the valence band in monolayer $\mathrm{MoS}_{2}{ }^{36-38}$. The $\mathrm{MoS}_{2} \mathrm{PL}$ emission is sensitive to the substrate. The PL emission intensity of $\mathrm{MoS}_{2}$ on $\mathrm{SiO}_{2}$ will be stronger than that of $\mathrm{MoS}_{2}$ on the TI film. As shown in Fig. $5 \mathrm{a}$, the intensities of the PL peaks drastically increase 

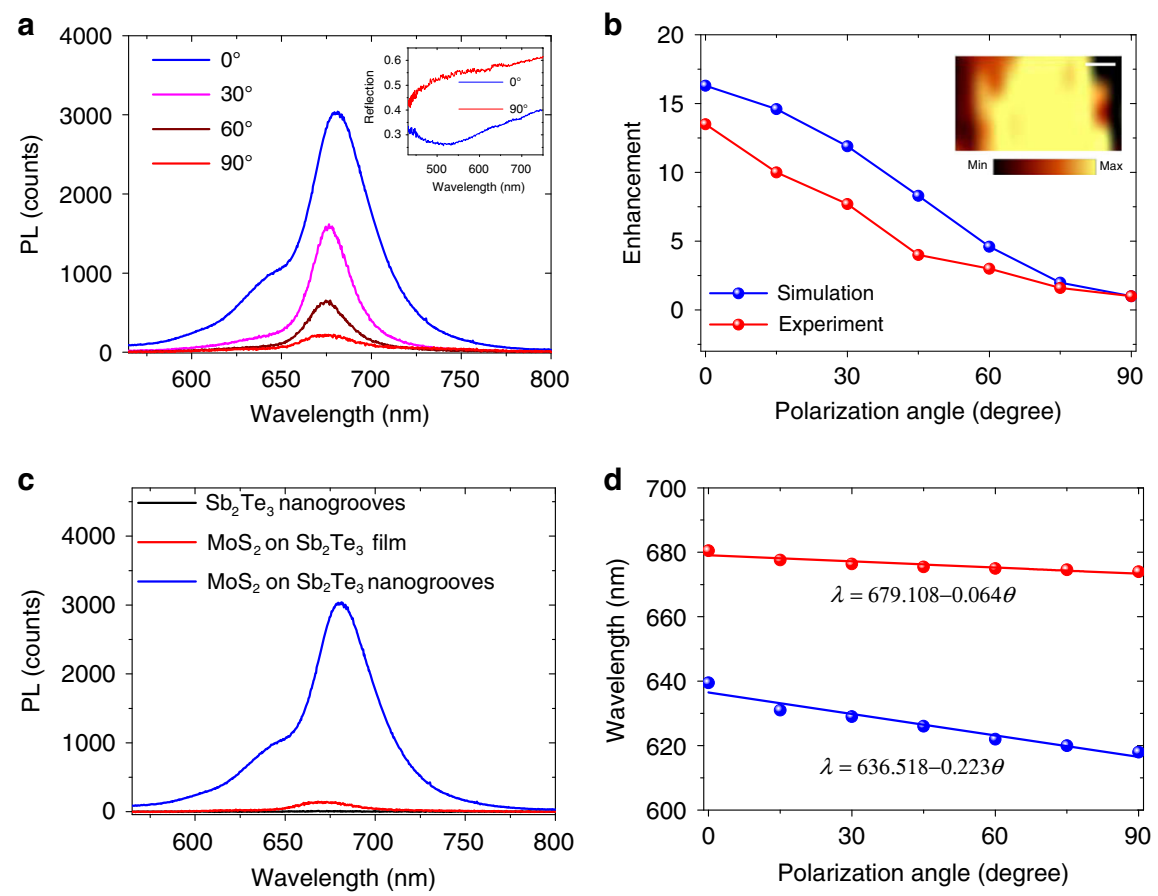

Fig. $5 \mathrm{PL}$ emission response of the monolayer $\mathrm{MoS}_{2} / \mathrm{Sb}_{2} \mathrm{Te}_{3}$ nanogroove heterostructure. a PL emission spectra of the monolayer $\mathrm{MoS}_{2} / \mathrm{Sb}_{2} \mathrm{Te} \mathrm{e}_{3}$ nanogroove heterostructure for different incident polarization angles $\theta$. The inset shows the reflection spectra of $\mathrm{Sb}_{2} \mathrm{Te}_{3}$ nanogrooves with monolayer $\mathrm{MoS}_{2}$ when $\theta=0^{\circ}$ and $90^{\circ}$. b Enhancement factors of the integrated electric field intensity in $\mathrm{Sb}_{2} \mathrm{Te}_{3}$ nanogrooves at $532 \mathrm{~nm}$ and $\mathrm{PL}$ emission at the $A$ excitonic peak of monolayer $\mathrm{MoS}_{2}$ for different $\theta$ compared to the case of $\theta=90^{\circ}$. The inset shows the PL intensity map spectrally integrated in the range of $665-695 \mathrm{~nm}$ when $\theta=0^{\circ}$ in the nanogroove area marked as the white dashed square frame in Fig. 4a. The scale bar is $1 \mu \mathrm{m}$. c PL emission spectra from the $\mathrm{Sb}_{2} \mathrm{Te}_{3}$ nanogrooves and monolayer $\mathrm{MoS}_{2}$ on the $\mathrm{Sb}_{2} \mathrm{Te}_{3}$ film/nanogrooves. $\mathbf{d}$ Dependence of $\mathrm{A}$ and $\mathrm{B}$ excitonic peak wavelengths on $\theta$.

with the decrease in $\theta$ from $90^{\circ}$ to $0^{\circ}$. The PL emission intensity with $\theta=0^{\circ}$ is particularly enhanced compared with the case of $\theta=90^{\circ}$. Actually, the PL emission improvement is mainly dependent on the electric field enhancement in luminescent materials ${ }^{21,31,37}$. To clarify the mechanism of PL reinforcement, we numerically calculate the integrated electric field intensity in the $\mathrm{Sb}_{2} \mathrm{Te}_{3}$ nanogrooves. In contrast with the electric field with $\theta=90^{\circ}$, we find that the enhancement factor of the integrated electric field intensity at $532 \mathrm{~nm}$ monotonically decreases as $\theta$ increases from $0^{\circ}$ to $90^{\circ}$. As shown in Fig. $5 \mathrm{~b}$, the enhancement factor of the electric field intensity is close to the reinforced strength of the $\mathrm{MoS}_{2} \mathrm{PL}$ excitonic peak. Therefore, the electric field enhancement plays a critical role in the PL reinforcement. In addition, the $\mathrm{MoS}_{2}$ PL emission is influenced by the support structures. The low reflection at the exciton wavelength may weaken the detected PL emission (Supplementary Fig. S8). Moreover, the PL peak wavelengths exhibit a linear redshift with decreasing $\theta$, as depicted in Fig. $5 \mathrm{~d}$. The shifts satisfy the relations $\lambda=679.108-0.064 \theta$ and $\lambda=$ 636.518- $0.223 \theta$ for the two PL peaks. When $\theta=0^{\circ}$, the PL peaks can approach the 680.5 and $638.0 \mathrm{~nm}$ wavelengths. Here, the redshift of the PL peak can be attributed to the generation of trions (a type of quasiparticle state) in monolayer $\mathrm{MoS}_{2}$ induced by the doping of MPR-excited hot electrons ${ }^{37,38}$. The A-trion, neutral A exciton, and $\mathrm{B}$ exciton in $\mathrm{MoS}_{2}$ can be extracted by fitting the PL spectrum using the multi-Lorentzian fitting method $^{38}$. The $\mathrm{MoS}_{2} \mathrm{~A}-$ trion, A exciton, and B exciton peaks approximately localize at $687.4,673.2$, and $618.0 \mathrm{~nm}$ (i.e., $1.80,1.84$, and $2.01 \mathrm{eV}$ ) in the heterostructure with $\theta=90^{\circ}$, respectively (Supplementary Fig. S7). The A exciton dominates the $\mathrm{MoS}_{2} \mathrm{PL}$ emission when $\theta=90^{\circ}$ and decays with decreasing $\theta$, while the $\mathrm{A}-$ trion increases gradually. This may stem from the higher hotelectron doping in $\mathrm{MoS}_{2}$ induced by the stronger magnetic resonance in the nanogrooves with smaller $\theta^{38}$. This phenomenon confirms the exciton-trion competition in plasmon systems ${ }^{37}$. The MPR-induced field enhancement and exciton-trion competition result in the different portions of the A exciton and A-trion/B exciton when $\theta$ changes from $90^{\circ}$ to $0^{\circ}$ (Supplementary Fig. S7). The appearance of hot electron-induced trions may also cause the reduction of the PL enhancement ${ }^{37}$. The energy shift of the A peak is $20 \mathrm{meV}$ when $\theta$ changes from $90^{\circ}$ to $0^{\circ}$, identical to the binding energy of trions in monolayer $\mathrm{MoS}_{2}{ }^{38}$. The dependence of the PL emission height and 
position on the incident polarization offers a controllable scheme for tailoring light- $\mathrm{MoS}_{2}$ interactions in artificial nanostructures. As depicted in Fig. 5c, the PL emission of monolayer $\mathrm{MoS}_{2}$ based on MPR shows a strong reinforcement of 21-fold, a remarkable value compared with that of EPRs on TI nanoplates ${ }^{21}$. It should be noted that the PL emission reinforcement mainly results from the enhancement of the integrated electric field intensity in the laser impingement area. Therefore, the reinforcement of the PL intensity is lower than that of the electric field in Fig. 2f. As shown in Fig. 4f, the Raman signal can be improved by one order of magnitude with the generation of magnetic resonance.

\section{Discussion}

In this article, the topological optical features of a $\mathrm{Sb}_{2} \mathrm{Te}_{3}$ single crystal have been experimentally demonstrated. The results show that the surface and bulk states of the $\mathrm{Sb}_{2} \mathrm{Te}_{3}$ TI exhibit obvious metal- and semiconductor-like characteristics in the range from UV to near-infrared, respectively, enabling plasmonic excitation at high frequencies. Visible range MPR-like magnetic resonances were first observed in $\mathrm{Sb}_{2} \mathrm{Te}_{3}$ nanogrooves nanofabricated using the FIB milling method, breaking through the research limitation of TI EPRs ${ }^{16-21}$. Both the experimental and simulation results indicate that this MPR response particularly depends on the structural parameters. More specifically, the MPR wavelength redshifts with increasing nanogroove height and pitch, while it presents a blueshift with increasing nanogroove width. The MPR behavior can be effectively analyzed by the MLC circuit theoretical model. To explore potential applications of this MPR effect, we have integrated an advanced 2D nanomaterial (i.e., monolayer $\mathrm{MoS}_{2}$ ) with TI nanostructures to boost the light-matter interactions as an example. The experimental results show that the PL emission of monolayer $\mathrm{MoS}_{2}$ can be dramatically reinforced with MPR generation, breaking the intrinsic limitation of the poor interaction between light and atomically thin materials. The peak intensities and wavelengths of the $\mathrm{MoS}_{2}$ PL can be tuned by adjusting the polarization angle of the incident light, which can be attributed to the resonanceinduced polarization-dependent electric field enhancement and generation of the A-trion. The $\mathrm{MoS}_{2} \mathrm{PL}$ emission can be reinforced by 21 -fold based on the MPRs in TI nanostructures, remarkably compared with TI-based EPRs ${ }^{21}$. The conventional Au material can also effectively promote the PL emission of $\mathrm{MoS}_{2}{ }^{39}$. TIs can support plasmons at wavelengths from $\mathrm{UV}$ to $\mathrm{THz}^{16-21}$, making the generation of electric/magnetic resonances in an ultrabroad range possible. The excited wavelengths for Au-based PL enhancement can be extended by TI-based plasmons. The resonant field enhancement in the TI nanogrooves may not be the highest value, but the resonance spectral width with strong field enhancement is particularly broad. When the resonance wavelength is located around the $\mathrm{MoS}_{2}$ PL peak position, the electric field at $532 \mathrm{~nm}$ can still be enhanced by $>100$ times, which results in obvious $\mathrm{MoS}_{2}$ PL reinforcement (Supplementary Fig. S9). We find that the field enhancement can be further promoted through structural modification (Supplementary Fig. S10), enabling more applications of TIs in optical devices, such as nanoscale light sources, nonlinear frequency converters, and lightharvesting elements. This kind of magnetic resonance can also be generated in other TIs, such as $\mathrm{Bi}_{2} \mathrm{Te}_{3}$ and $\mathrm{Bi}_{2} \mathrm{Se}_{3}$. The various structures, such as nanopillars ${ }^{6}$, nanoclusters ${ }^{7}$, nanoparticles on film ${ }^{8,9}$, and nanocups ${ }^{10}$, will further enrich MPR effects in TI systems. These results not only enrich TI optical physics but also advance applications of TIs in optoelectronic devices and artificial magnetic materials.

\section{Materials and methods}

\section{Growth of the $\mathrm{Sb}_{2} \mathrm{Te}_{3}$ single crystal}

The high-quality $\mathrm{Sb}_{2} \mathrm{Te}_{3}$ single crystal is grown using the melting and slow-cooling method. High-purity Sb and Te powders with an atomic ratio of $2: 3$, as the starting materials, are sealed in a quartz tube. The crystals can be grown in a vertical furnace according to the following procedures: (1) The $\mathrm{Sb}$ and Te mixed powders are heated to $900{ }^{\circ} \mathrm{C}$ and completely melt. (2) The temperature is reduced quickly to $650{ }^{\circ} \mathrm{C}$ at a rate of $60^{\circ} \mathrm{C} / \mathrm{h}$ and then slowly to $550{ }^{\circ} \mathrm{C}$ at a rate of $2{ }^{\circ} \mathrm{C} / \mathrm{h}$. (3) The mixture is naturally cooled to room temperature. The $\mathrm{Sb}_{2} \mathrm{Te}_{3}$ singlecrystalline character and stoichiometry can be confirmed by TEM, Raman, and EDS characterization.

Fabrication of $\mathrm{Sb}_{2} \mathrm{Te}_{3}$ nanostructures and transfer of $\mathrm{MoS}_{2}$ The $\mathrm{Sb}_{2} \mathrm{Te}_{3}$ film is mechanically exfoliated on a $\mathrm{Si}$ substrate using "Scotch" tape from the $\mathrm{Sb}_{2} \mathrm{Te}_{3}$ single crystal. The nanogroove grating is fabricated on the $\mathrm{Sb}_{2} \mathrm{Te}_{3}$ film using a FIB milling system (FEI Helios G4 $\mathrm{CX}$ ) with a $30 \mathrm{kV}$ voltage and a $7.7 \mathrm{pA}$ current. The beam current should be controlled at a relatively low level for robust FIB fabrication. The $\mathrm{MoS}_{2}$ flakes are fabricated by exfoliating them from the $\mathrm{MoS}_{2}$ bulk material, repeatedly peeling them off with tape, and sticking them on a polydimethylsiloxane (PDMS) film. If a $\mathrm{MoS}_{2}$ flake on the PDMS has the most transparent area of several microns in size, then the $\mathrm{MoS}_{2}$ flake is transferred onto the $\mathrm{Sb}_{2} \mathrm{Te}_{3}$ nanogrooves using an optical microscope/micromanipulation system. Thus, the dry fixed-point transfer of $\mathrm{MoS}_{2}$ is completed.

\section{Characterization of materials and nanostructures}

The electron diffraction pattern and high-resolution TEM image of the $\mathrm{Sb}_{2} \mathrm{Te}_{3}$ single crystal are obtained by TEM equipment (FEI Talos F200X) with a voltage of $200 \mathrm{kV}$. Raman spectra of $\mathrm{MoS}_{2}$ and $\mathrm{Sb}_{2} \mathrm{Te}_{3}$, as well as PL emission spectra, are acquired using confocal micro-spectrometry 
(WITec Alpha 300R) with a linearly polarized $532 \mathrm{~nm}$ laser and an adjustable beam size (minimum diameter: $400 \mathrm{~nm}$ ). SEM images of $\mathrm{Sb}_{2} \mathrm{Te}_{3}$ nanostructures are acquired by SEM equipment integrated with the FIB (FEI Helios G4 CX) using a $5 \mathrm{kV}$ voltage and a $21 \mathrm{pA}$ current. To avoid damaging the TI material, the beam current should not be too high $(<43 \mathrm{pA})$ for SEM imaging. AFM images and height profiles of $\mathrm{Sb}_{2} \mathrm{Te}_{3}$ nanostructures are obtained by a commercial AFM system (Bruker). The relative permittivity of the $\mathrm{Sb}_{2} \mathrm{Te}_{3}$ single crystal in the UV, visible and nearinfrared ranges is measured by a spectroscopic ellipsometer (HORIBA) with an angle of $70^{\circ}$ for incident light. Reflection spectra from $\mathrm{Sb}_{2} \mathrm{Te}_{3}$ nanogrooves with/without $\mathrm{MoS}_{2}$ are measured by a home-made micro-spectrometer with a white light source impinging on the sample through a microscope and then reflected onto a CCD camera with a spectrometer (Andor).

\section{Numerical simulations}

The reflection spectra and field distributions of $\mathrm{Sb}_{2} \mathrm{Te}_{3}$ nanostructures are numerically simulated using the FDTD method $^{20,40,41}$. The perfectly matched layer absorbing boundary condition and periodic boundary condition are set at the top/bottom and left/right sides of the computational space, respectively. A non-uniform mesh is employed in the $x$ and $z$ axis directions of TI nanostructures. The maximum mesh steps of the $\mathrm{Sb}_{2} \mathrm{Te}_{3}$ surface layers and monolayer $\mathrm{MoS}_{2}(0.615 \mathrm{~nm})$ are set as $0.3 \mathrm{~nm}$ and $0.1 \mathrm{~nm}$, respectively. The maximum mesh step of the $\mathrm{Sb}_{2} \mathrm{Te}_{3}$ bulk layer, air, and Si substrate is set as $5 \mathrm{~nm}$. The relative permittivities in Fig. 1e, $\mathrm{f}$ are set for the surface and bulk states of the $\mathrm{Sb}_{2} \mathrm{Te}_{3} \mathrm{TI}$, respectively. The complex relative permittivity of the Si substrate is achieved from experimental data ${ }^{42}$. The relative permittivity of monolayer $\mathrm{MoS}_{2}$ measured by $\mathrm{Li}$ et al. is used in the simulations ${ }^{43}$. The reflection spectra are calculated using $R=\left|P_{\mathrm{r}} / P_{\mathrm{i}}\right|$, where $P_{\mathrm{i}}$ and $P_{\mathrm{r}}$ are the light powers incident on and reflected from the $\mathrm{Sb}_{2} \mathrm{Te}_{3}$ nanostructures, respectively.

\section{Acknowledgements \\ This work was supported by the National Key R\&D Program of China (2017YFA0303800), National Natural Science Foundation of China (11974283, 61705186, 11774290, 11634010, and 61605065), Natural Science Basic Research Plan in Shaanxi Province of China (2020JM-130), Guangzhou Science and Technology Program (201804010322), and Guangdong Basic and Applied Basic Research Foundation (2020A1515011510). We thank the Analytical \& Testing Center of Northwestern Polytechnical University (NPU) for the FIB nanofabrication as well as AFM, SEM, and TEM measurements. We also thank Dr. C. Liu at NPU and Prof. X. Li at Westlake University for help with the TEM measurement and FIB fabrication.}

\section{Author details}

${ }^{1}$ MOE Key Laboratory of Material Physics and Chemistry under Extraordinary Conditions, and Shaanxi Key Laboratory of Optical Information Technology, School of Physical Science and Technology, Northwestern Polytechnical University, 710129 Xi'an, China. ${ }^{2}$ Institute for Superconducting \& Electronic Materials and ARC Centre of Excellence in Future Low-Energy Electronics, University of Wollongong, North Wollongong, NSW 2500, Australia. ${ }^{3}$ Center for Artificial-Intelligence Nanophotonics, School of Optical-Electrical and
Computer Engineering, University of Shanghai for Science and Technology, 200093 Shanghai, China. ${ }^{4}$ Guangdong Provincial Key Laboratory of Optical Fiber Sensing and Communications, Institute of Photonics Technology, Jinan University, 510632 Guangzhou, China. ${ }^{5}$ State Key Laboratory of Solidification Processing, School of Materials Science and Engineering, Northwestern Polytechnical University, 710072 Xi'an, China

\section{Author contributions}

H.L. conceived the idea, carried out numerical simulations, theoretical calculations, structure fabrication, material/structure characterization and analysis of results, drew the figures, and wrote the manuscript text. Y.Z. conducted the reflection spectra measurements and discussed the results. Z.Y. and W.Z. fabricated the $\mathrm{Sb}_{2} \mathrm{Te}_{3}$ single crystal and discussed the characterization. Y.L. participated in the fabrication and characterization of nanostructures. M.Z. conducted the exfoliation and transfer of $M_{2} S_{2}$. W.Z. took part in the measurement of the $\mathrm{Sb}_{2} \mathrm{Te}_{3}$ relative permittivity. X.G., D.M., F.X., T.M., X.W., M.G., and J.Z. discussed the results and promoted the manuscript presentation. All authors substantially contributed to the manuscript.

\section{Data availability}

The data sets generated and analyzed in the article are available from the corresponding authors upon reasonable request.

\section{Conflict of interest}

The authors declare that they have no conflict of interest.

Supplementary information is available for this paper at https:/doi.org/ 10.1038/541377-020-00429-X.

Received: 2 June 2020 Revised: 30 October 2020 Accepted: 6 November 2020

Published online: 23 November 2020

\section{References}

1. Smith, D. R., Pendry, J. B. \& Wiltshire, M. C. K. Metamaterials and negative refractive index. Science 305, 788-792 (2004).

2. Ma, H. F. \& Cui, T. J. Three-dimensional broadband ground-plane cloak made of metamaterials. Nat. Commun. 1, 21 (2010).

3. Yen, T. J. et al. Terahertz magnetic response from artificial materials. Science 303, 1494-1496 (2004).

4. Linden, $\mathrm{S}$. et al. Magnetic response of metamaterials at 100 Terahertz. Science 306, 1351-1353 (2004).

5. Zhou, J. et al. Saturation of the magnetic response of split-ring resonators at optical frequencies. Phys. Rev. Lett. 95, 223902 (2005).

6. Grigorenko, A. N. et al. Nanofabricated media with negative permeability at visible frequencies. Nature 438, 335-338 (2005).

7. Shafiei, F. et al. A subwavelength plasmonic metamolecule exhibiting magnetic-based optical Fano resonance. Nat. Nanotechnol. 8, 95-99 (2013).

8. Liu, N. et al. Infrared perfect absorber and its application as plasmonic sensor. Nano Lett. 10, 2342-2348 (2010).

9. Chen, S. et al. Plasmon-induced magnetic resonance enhanced Raman spectroscopy. Nano Lett. 18, 2209-2216 (2018).

10. Ding, S. J. et al. Magnetic plasmon-enhanced second-harmonic generation on colloidal gold nanocups. Nano Lett. 19, 2005-2011 (2019).

11. Papasimakis, N. et al. The magnetic response of graphene split-ring metamaterials. Light Sci. Appl. 2, e78 (2013).

12. König, M. et al. Quantum spin Hall insulator state in $\mathrm{HgTe}$ quantum wells. Science 318, 766-770 (2007).

13. Zhang, H. J. et al. Topological insulators in Bi2Se3 Bi2Te3 Sb2Te3 with a single Dirac cone on the surface. Nat. Phys. 5, 438-442 (2009).

14. Hasan, M. Z. \& Kane, C. L. Colloquium: topological insulators. Rev. Mod. Phys. 82, 3045-3067 (2010)

15. Yue, Z. J. et al. Nanometric holograms based on a topological insulator material. Nat. Commun. 8, 15354 (2017).

16. Di Pietro, P. et al. Observation of Dirac plasmons in a topological insulator. Nat. Nanotechnol. 8, 556-560 (2013).

17. $\mathrm{Ou}, \mathrm{J} . \mathrm{Y}$. et al. Ultraviolet and visible range plasmonics in the topological insulator Bi1.5Sb0.5Te1.8Se1.2. Nat. Commun. 5, 5139 (2014). 
18. Zhao, M. et al. Visible surface plasmon modes in single Bi2Te3 nanoplate. Nano Lett. 15, 8331-8335 (2015).

19. Sim, S. et al. Ultra-high modulation depth exceeding $2,400 \%$ in optically controlled topological surface plasmons. Nat. Commun. 6, 8814 (2015).

20. Yue, Z. J. et al. Intrinsically core-shell plasmonic dielectric nanostructures with ultrahigh refractive index. Sci. Adv. 2, e1501536 (2016).

21. Zhao, M. et al. Actively tunable visible surface plasmons in Bi2Te3 and their energy-harvesting applications. Adv. Mater. 28, 3138-3144 (2016).

22. Tang, F. et al. Comprehensive search for topological materials using symmetry indicators. Nature 566, 486-489 (2019).

23. Vergniory, M. G. et al. A complete catalogue of high-quality topological materials. Nature 566, 480-485 (2019).

24. Barnes, W. L., Dereux, A. \& Ebbesen, T. W. Surface plasmon subwavelength optics. Nature 424, 824-830 (2003).

25. Ding, K. \& Ning, C. Z. Metallic subwavelength-cavity semiconductor nanolasers. Light Sci. Appl. 1, e20 (2012).

26. Ren, M. X. et al. Linearly polarized light emission from quantum dots with plasmonic nanoantenna arrays. Nano Lett. 15, 2951-2957 (2015).

27. Jiang, Y. P. et al. Landau quantization and the thickness limit of topological insulator thin films of Sb2Te3. Phys. Rev. Lett. 108, 016401 (2012).

28. Xia, B. et al. Indications of surface- dominated transport in single crystalline nanoflake devices of topological insulator Bi1.5Sb0.5Te1.8Se1.2. Phys. Rev. B 87, 085442 (2013)

29. Sarychev, A. K., Shvets, G. \& Shalaev, V. M. Magnetic plasmon resonance. Phys. Rev. E 73, 036609 (2006).

30. Guo, Y. M., Shuai, Y. \& Tan, H. P. Mechanism of polaritons coupling from perspective of equivalent MLC circuits model in slit arrays. Opt. Express 27, 21173-21184 (2019).
31. Xia, F. N. et al. Two-dimensional material nanophotonics. Nat. Photonics 8 , 899-907 (2014).

32. Säynätjoki, A. et al. Ultra-strong nonlinear optical processes and trigonal warping in MoS2 layers. Nat. Commun. 8, 893 (2017).

33. Nan, H. Y. et al. Strong photoluminescence enhancement of MoS2 through defect engineering and oxygen bonding. ACS Nano 8, 5738-5745 (2014).

34. Liu, Y. L. et al. Layer-by-layer thinning of MoS2 by plasma. ACS Nano 7, 4202-4209 (2013).

35. Liao, F. et al. Enhancing monolayer photoluminescence on optical micro/ nanofibers for low-threshold lasing. Sci. Adv. 5, eaax7398 (2019).

36. Splendiani, A. et al. Emerging photoluminescence in monolayer MoS2. Nano Lett. 10, 1271-1275 (2010).

37. Li, J. et al. Tuning the photo-response in monolayer MoS2 by plasmonic nanoantenna. Sci. Rep. 6, 23626 (2016)

38. Mak, K. F. et al. Tightly bound trions in monolayer MoS2. Nat. Mater. 12 207-211 (2013).

39. Zeng, Y. et al. Highly enhanced photoluminescence of monolayer MoS2 with self-assembled Au nanoparticle arrays. Adv. Mater. Interfaces 4, 1700739 (2017).

40. Zhang, Y. N. et al. Full-visible multifunctional aluminium metasurfaces by in situ anisotropic thermoplasmonic laser printing. Nanoscale Horiz. 4, 601-609 (2019).

41. Taflove, A. \& Hagness, S. Computational Electrodynamics: The Finite-Difference Time-Domain Method 2nd edn (Artech House, Boston, 2000).

42. Palik, E. D. Handbook of Optical Constants of Solids 151-166 (Academic Press, New York, 1991).

43. Li, Y. L et al. Measurement of the optical dielectric function of monolayer transition-metal dichalcogenides: MoS2, MoSe2, WS2, and WSe2. Phys. Rev. B 90, $205422(2014)$ 